\title{
La anticoncepción en el siglo XXI
}

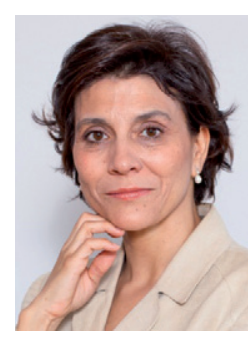

Pilar Lafuente González Unidad de Patología Uterina. Servicio de Ginecología y Obstetricia.

Hospital Universitario La Paz. Madrid.

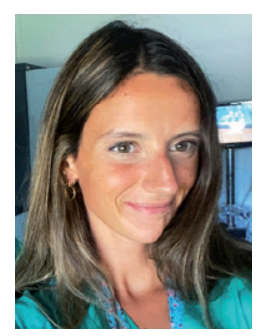

María Carbonell López Residente de ginecología y obstetricia.

Servicio de Ginecología y Obstetricia.

Hospital Universitario La Paz. Madrid.

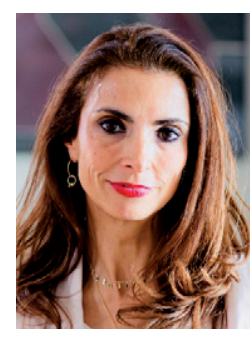

María de la Calle Fernández-Miranda Jefe de la Sección de Tocología de Alto Riesgo y Obstetricia Médica.

Servicio de Ginecología y Obstetricia. Hospital Universitario La Paz. Madrid.

\section{INTRODUCCIÓN}

La entrada del primer anticonceptivo combinado oral en la farmacopea de la ginecología fue en el año 1960, lo que supuso un hecho histórico sin precedentes, tanto desde el punto vista de la sexualidad (al separar la función reproductiva de la sexual), como desde la perspectiva médica, ya que se empezó a disponer de tratamiento para múltiples enfermedades ginecológicas (sangrado menstrual abundante, dismenorrea, irregularidades menstruales, etc.), hasta ese momento, inimaginable.

Han pasado más de 60 años desde entonces y, gracias al esfuerzo investigador de diferentes grupos de trabajo y de las empresas farmacéuticas, estamos consiguiendo formulaciones con dosis cada vez menores de estrógenos sintéticos $(<35 \mu \mathrm{g})$, con estrógenos naturales y con gestágenos con un perfil menos androgénico (gestodeno, dienogest, drospirenona) en dosis más bajas, que hacen de los diversos anticonceptivos que han ido surgiendo en los últimos años verdaderas dianas terapéuticas, con menos efectos secundarios. También se han desarrollado diferentes vías de administración (vaginal, subcutánea, transdérmica, intrauterina...), que ayudan a la cumplimentación por parte de la paciente, generando adherencia al tratamiento y, por lo tanto, aumentado la eficacia del fármaco; así como nuevas pautas de administración $(24+4 ; 26+2$; pauta extendida y continua) (fig. 1) ${ }^{1,2}$. La anticoncepción del siglo XXI es un traje a medida de nuestras pacientes, de su situación personal y laboral, de su proyecto de vida y de su salud individual.

\section{CLASIFICACIÓN DE LOS MÉTODOS ANTICONCEPTIVOS Y SU MECANISMO DE ACCIÓN}

Los métodos anticonceptivos (MAC) son cualquier método, fármaco o dispositivo con el que se intenta 
Preparados monofásicos

21/7 (Pauta cíclica)

\begin{tabular}{|l|l|l|l|l|l|l|l|l|l|l|l|l|l|l|l|l|l|l|l|l|l|l|l|l|}
\hline & & & & & & & & & & & & & & & & & & & & & & & & \\
\hline
\end{tabular}

24/4 (Pauta cíclica)

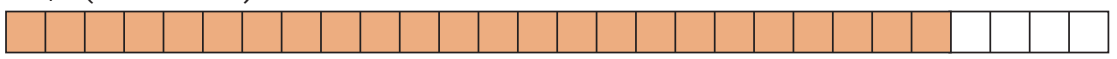

84/7 (Pauta extendida)

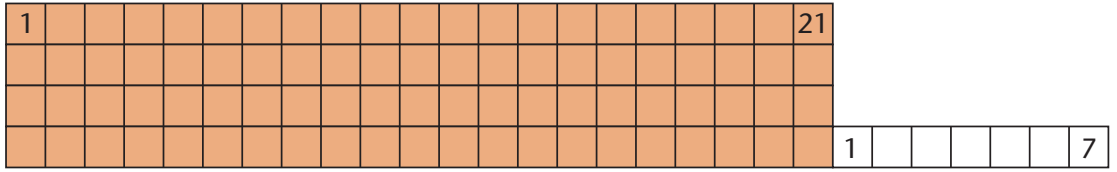

Pauta continua

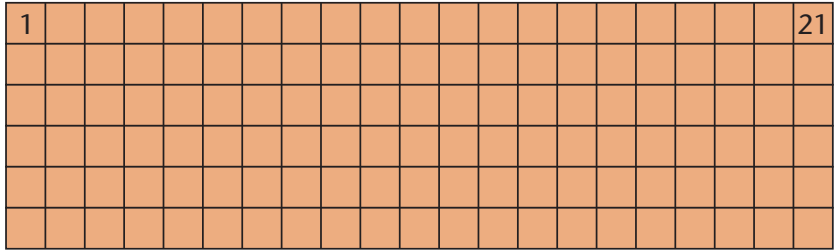

Con principio activo combinado

Sin principio activo

Figura 1. Preparados monofásicos, que contienen la misma cantidad de estrógenos y progestinas en todas las pastillas. Puede cambiar el número de días con placebo de 7 a 4, mostrando una mayor eficacia anticonceptiva, al impedir la elevación de la hormona foliculoestimulante en estos días de descanso. La pauta extendida $(21 \times 4)$ 84/7 y la pauta continua aportan ventajas tanto anticonceptivas como de control de la sintomatología ginecológica (dismenorrea, sangrado abundante, etc.) y menores variaciones cíclicas de los parámetros metabólicos plasmáticos. Los preparados fásicos fueron diseñados para reducir la cantidad total de progestinas por ciclo sin sacrificar la eficacia anticonceptiva ni la regulación del ciclo. Hoy se encuentran prácticamente en desuso por no aportar dichos beneficios².

prevenir el embarazo. Los MAC pueden clasificarse teniendo en cuenta diferentes aspectos (tabla 1$)^{1-5}$.

\section{COMPONENTE ESTROGÉNICO}

Los anticonceptivos hormonales combinados (AHC) pueden contener dos tipos de estrógenos: el etinilestradiol (EE) (dosis entre 15 y $40 \mu \mathrm{g}$ ) es un estrógeno sintético, muchísimo más potente que los estrógenos naturales (valerianato de estradiol y hemihidrato de estradiol). Los estrógenos naturales tienen un impacto más favorable en el perfil lipídico y en los parámetros de la coagulación y, por lo tanto, menor incidencia de enfermedad tromboembólica (ETE).
La función del componente estrogénico en los AHC es estabilizar el endometrio, evitar sangrados irregulares (principal efecto secundario de los anticonceptivos con solo gestágenos [ASG]) e inhibir la ovulación, actuando sobre el eje hipotálamohipofisario para bloquear la secreción de hormona foliculoestimulante (FSH; del inglés, follicle-stimulating hormone) y de hormona luteinizante (LH; del inglés, luteinizing hormone). Suprimen también la producción androgénica a nivel ovárico ${ }^{6}$. Y, asimismo, disminuye la cantidad de testosterona libre en sangre, al aumentar la síntesis hepática de la globulina de fijación a las hormonas sexuales (SHBG; del inglés, sex hormone-binding globulin), posible marcador indirecto del riesgo de $\mathrm{ETE}^{7}$. 
Tabla 1. Clasificación de los métodos anticonceptivos teniendo en cuenta diferentes mecanismos de acción y características del método anticonceptivo

\begin{tabular}{|c|c|c|}
\hline Criterios de clasificación & Mecanismo de acción (MA) & Tipos \\
\hline Métodos reversibles & $\begin{array}{c}\text { Capacidad de recuperar la fertilidad } \\
\text { al suspender el MAC }\end{array}$ & $M N, M N H, M B, M H, L A R C$ \\
\hline Métodos irreversibles & No se recupera la fertilidad posteriormente & $\begin{array}{l}\text { Ligadura de trompas } \\
\text { Vasectomía }\end{array}$ \\
\hline Métodos naturales (MN) & $\begin{array}{c}\text { Evitar relaciones sexuales en el período } \\
\text { fértil del ciclo }\end{array}$ & $\begin{array}{l}\text { Método del ritmo (Ogino-Knaus) } \\
\text { Método de la temperatura basal } \\
\text { Método de Billings } \\
\text { Método sintotérmico } \\
\text { Amenorrea de la lactancia (MELA) }\end{array}$ \\
\hline $\begin{array}{l}\text { Métodos no hormonales } \\
(\mathrm{MNH})\end{array}$ & $\begin{array}{c}\text { MA múltiple: impiden la fecundación, } \\
\text { son tóxicos para el espermatozoide } \\
\text { y para el óvulo mediante una reacción } \\
\text { a cuerpo extraño }\end{array}$ & DIU-Cu \\
\hline Métodos de barrera (MB) & $\begin{array}{l}\text { Evitan que los espermatozoides lleguen } \\
\text { al canal cervical }\end{array}$ & $\begin{array}{c}\text { Preservativo masculino y femenino } \\
\text { Cremas espermicidas } \\
\text { Diafragma, esponjas, capuchón } \\
\text { Marcha atrás }\end{array}$ \\
\hline Métodos hormonales (MH) & \multirow{3}{*}{$\begin{array}{c}\text { Inhiben la ovulación } \\
\text { Desencadenan espesamiento del moco } \\
\text { cervical } \\
\text { Disminuyen la motilidad tubárica } \\
\text { y espermática } \\
\text { Provocan adelgazamiento del endometrio }\end{array}$} & Todos los que contienen hormonas \\
\hline \multirow{2}{*}{$\begin{array}{l}\text { Atendiendo al componente } \\
\text { hormonal }\end{array}$} & & $\begin{array}{c}\text { ASG: oral, en inyección } \\
\text { (Depo-Progevera), implante, } \\
\text { DIU-LNG }\end{array}$ \\
\hline & & $\begin{array}{l}\text { AHC (estrógeno + progesterona): } \\
\text { oral, vaginal, en parches }\end{array}$ \\
\hline Eficacia real del método & Cada MAC tiene su MA & Figura $2^{4,5}$ \\
\hline $\begin{array}{l}\text { Métodos de larga duración } \\
\text { (LARC) }\end{array}$ & & $\begin{array}{c}\text { Implante subdérmico } \\
\text { DIU-LNG } \\
\text { DIU-Cu }\end{array}$ \\
\hline \multirow{2}{*}{$\begin{array}{l}\text { Anticoncepción } \\
\text { de emergencia }\end{array}$} & Retrasa la ovulación & $\begin{array}{l}1,5 \mathrm{~g} \text { de levonorgestrel } \\
30 \mathrm{mg} \text { de acetato de ulipristal }\end{array}$ \\
\hline & Impide la fecundación y anidación & DIU-Cu (300); DIU-LNG \\
\hline
\end{tabular}

AHC: anticoncepción hormonal combinada; ASG: anticoncepción con solo gestágenos; DIU-Cu: dispositivo intrauterino de cobre; DIU-LNG: dispositivo intrauterino liberador de levonorgestrel; LARC: anticoncepción reversible de larga duración (long-acting reversible contraception); MAC: métodos anticonceptivos; MELA: método (anticonceptivo) de lactancia (materna) y amenorrea.

Los AHC actuales son de baja dosis $(\mathrm{EE}<50 \mu \mathrm{g})$ y, por lo tanto, el efecto de ETE estaría más relacionado con la progestina (PG) utilizada ${ }^{7}$ (potencia antiandrogénica y de tercera generación).

\section{PROGESTINAS}

Se denomina PG a los gestágenos sintéticos que se utilizan en los preparados anticonceptivos ${ }^{8}$. Las PG tienen diferentes propiedades farmacológicas 


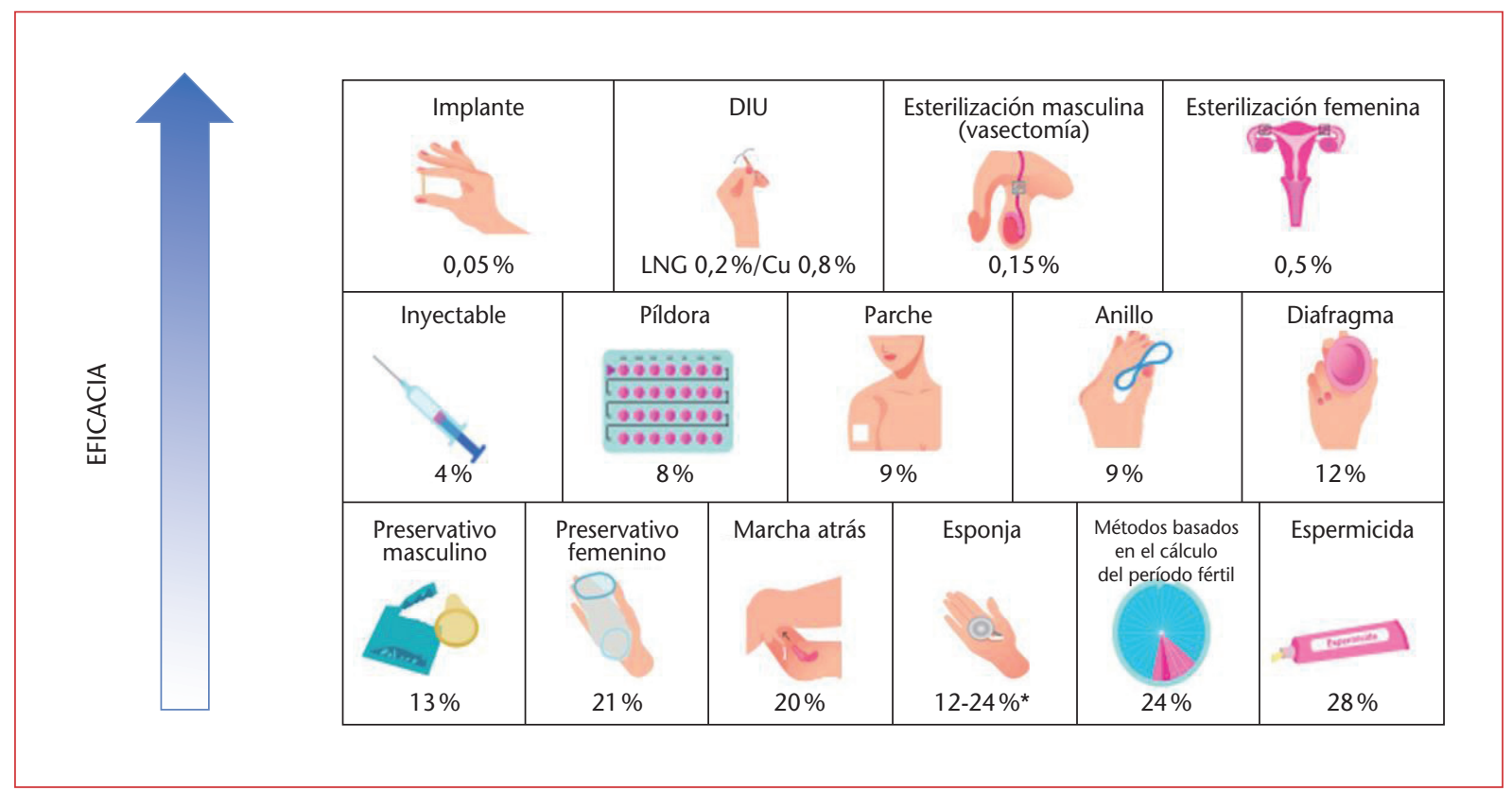

Figura 2. Clasificación de los diferentes métodos anticonceptivos atendiendo a la eficacia real del método. Es muy importante a la hora de asesorar en materia anticonceptiva ${ }^{4,5}$.

*Del $12 \%$ en nulíparas y del $24 \%$ en multíparas.

Cu: cobre; DIU: dispositivo intrauterino; LNG: levonorgestrel.

dependiendo de la molécula madre de la que derivan: testosterona, progesterona o espironolactona. Y se clasifican en PG de primera y segunda generación las sintetizadas antes de la década de 1980, y las nuevas PG de tercera y cuarta generación, de las décadas de 1990 y 2000. Las PG, además de unirse a los receptores de la progesterona, se pueden unir a los receptores de estrógenos, andrógenos, mineralocorticoides y glucocorticoides. Las nuevas PG han sido diseñadas para no tener acción androgénica o estrogénica y, así, presentar una actividad más cercana a la hormona fisiológica, lo que disminuye considerablemente los efectos secundarios (acné, hinchazón, aumento de peso, irritabilidad, retención de líquidos, disminución de la libido, sangrados irregulares... $)^{8}$.

Este componente de los AHC tiene como misión principal inhibir la ovulación, al impedir el pico de la LH, pero también produce espesamiento del moco cervical, adelgaza la línea endometrial y altera la secreción y la motilidad de las trompas ${ }^{1}$.

\section{BENEFICIOS NO ANTICONCEPTIVOS DE LOS ANTICONCEPTIVOS}

Los AHC mejoran tanto la dismenorrea (todos) como el síndrome premenstrual (drospirenona en pauta de $24+4$ ), disminuyen la cantidad del sangrado menstrual (dienogest) y, por lo tanto, mejoran y previenen la anemia ferropénica. También ejercen protección frente a la enfermedad inflamatoria pélvica; disminuyen el dolor asociado a la endometriosis (dienogest); poseen efectos beneficiosos sobre la piel: acné, hirsutismo, seborrea y alopecia (clormadinona, drospirenona, dienogest, ciproterona); tienen efectos beneficiosos sobre el hueso (excepto el acetato de medroxiprogesterona -Depo-Progevera-); reducen la incidencia de quistes funcionales en los ovarios y de la enfermedad benigna de la mama, y disminuyen la incidencia de cáncer de ovario, de endometrio y de colon, así como el embarazo ectópico.

Los ASG se utilizan para el tratamiento del sangrado menstrual abundante (dispositivo intraute- 
rino liberador de levonorgestrel [DIU-LNG]) y de la hiperplasia de endometrio (DIU-LNG), y previenen el cáncer de endometrio en las pacientes con anovulación crónica y sobrepeso importante (DIU-LNG). Están indicados en las pacientes que tienen contraindicado el uso de estrógenos.

El DIU de cobre (DIU-Cu) es protector frente al cáncer de cérvix, así como frente al cáncer de endometrio. Y, por último, el preservativo masculino y femenino previene las enfermedades de transmisión sexual.

\section{VALORACIÓN PREVIA A LA INDICACIÓN DE ANTICONCEPTIVOS}

Es imprescindible una historia clínica completa (en la que se descarten los factores de riesgo de ETE) y centrada en las necesidades y particularidades de cada paciente. Se debe realizar el cálculo del índice de masa corporal, así como la toma de la tensión arterial. La exploración ginecológica previa (genital y mamaria), las determinaciones analíticas y la realización de una citología cervicovaginal se consideran recomendables, pero no de obligado cumplimiento.

Posteriormente, se realizará una visita a los 3-6 meses de haber iniciado el MAC, para preguntar sobre la tolerancia, resolver dudas y mejorar la adherencia al tratamiento. En el caso de haber insertado un DIU, se hará una ecografía de control. El resto de los exámenes de salud se efectuarán como en el caso de cualquier otra mujer que no utilice MAC (citología, mamografía...).

\section{EFECTOS SECUNDARIOS DE LOS ANTICONCEPTIVOS HORMONALES COMBINADOS}

La enfermedad tromboembólica venosa (ETV) es uno de los efectos secundarios más graves en las mujeres que toman AHC (ocurre en el primer año de uso, con una incidencia máxima en los tres primeros meses). Es una entidad rara en jóvenes, excepto en el embarazo, que se incrementa indepen- dientemente de la edad de la mujer con una incidencia de 29 casos por 10000 mujeres/año, mientras que, en la población femenina general no embarazada, ocurre en 2 casos por cada $10000 \mathrm{mu}$ jeres/año. Habrá que descartar otros factores de riesgo asociados a la ETV, que incrementan la posibilidad de aparición de ETV antes de la prescripción, y desaconsejar su uso en estas pacientes (hábito tabáquico activo y mas de 35 años, obesidad, trombofilia hereditaria o adquirida, antecedente personal de trombosis venosa profunda ${ }^{6}$.

El riesgo más bajo para ETV es para los AHC que contienen levonorgestrel, norgestimato o noretisterona (5-7 casos de TEV por cada 10000 mujeres que los utilizan/año). Existe un riesgo intermedio para los que contienen etonogestrel y norelgestromina (6-12 casos anualmente por cada $10000 \mathrm{mu}-$ jeres). Mientras que el riesgo es mayor con gestodeno, desogestrel y drospirenona (9-12 casos anualmente por cada 10000 mujeres $)^{9}$.

Con los AHC $<35 \mu \mathrm{g}$, el riesgo de presentar trombosis arteriales, en mujeres sanas, es muy bajo (3 casos por 100000 habitantes/año) ${ }^{6}$. Pero, si estas mujeres fuman, el riesgo se multiplica por 14; si tienen HTA, el riesgo se incrementa por 6; si tienen un perfil lipídico adverso, el riesgo se incrementa por 25 y, si tienen diabetes, el riesgo se incrementa por $17^{6}$.

La hipertensión arterial es otro efecto secundario mayor, poco frecuente y que aparece en los seis primeros meses de uso.

Entre los efectos adversos menores, se encuentran: náuseas y vómitos, aumento de peso, mastodinia, cloasma, acné (con progestinas androgénicas), alteraciones del estado ánimo, colelitiasis, infecciones del tracto urinario inferior, disminución de la libido y sangrados intermenstruales (en los seis primeros meses de uso).

Los AHC tienen un papel protector (contra el cáncer de ovario, de endometrio y de colon). Se ha observado, no obstante, un incremento mínimo de los casos de cáncer de cérvix (riesgo relativo [RR]: 1,3-1,8) y de mama (RR: 1,24 en usuarias que iniciaron el tratamiento antes de los 20 años de edad y durante más de cinco años). 


\section{EFECTOS SECUNDARIOS DE OTROS MÉTODOS ANTICONCEPTIVOS}

Los ASG (tanto por vía oral como subdérmica o intrauterina) presentan alteración del patrón de sangrado (de lo que se deberá advertir antes de prescribirlos para disminuir la tasa de abandono) $y$, con menos frecuencia, cefaleas, quistes funcionales a nivel ovárico, acné, cambios del humor y mastodinia. El DIU-Cu puede producir sangrado abundante, anemia y dismenorrea ${ }^{1}$.

\section{PANDEMIA DE LA COVID-19 Y USO DE ANTICONCEPTIVOS}

Las mujeres que ya vienen utilizando un MAC, estén satisfechas y tengan adherencia al tratamiento no deben suspenderlo con el único pretexto de disminuir el riesgo de ETE durante el tiempo que dure la pandemia de la enfermedad por coronavirus de 2019 (COVID-19; del inglés, coronavirus disease 2019$)^{10,11}$. Se sabe que los estrógenos ejercen un efecto beneficioso en la respuesta inmunitaria frente a la COVID-19, con mayor producción de anticuerpos y mayor activación de la inmunidad celular ${ }^{12}$. Así, la suspensión del AHC podría acelerar la progresión de la COVID-1911. En el supuesto de que las pacientes en tratamiento con ACH se infecten por coronavirus y sean asintomáticas o paucisintomáticas, se añadirá heparina de bajo peso molecular durante el tiempo de infección y mientras se prolongue la inmovilidad ${ }^{10,11}$.

\section{BIBLIOGRAFÍA}

1. Percy L, Mansour D. Contraception made easy. Banbury: Scion Publishing Ltd; 2015.

2. Christin-Maitre S. History of oral contraceptive drugs and their use worldwide. Best Pract Res Clin Endocrinol Metab. 2013; 27(1):3-12.

3. Festin MPR. Overview of modern contraception. Best Pract Res Clin Obstet Gynaecol. 2020;66:4-14.

4. Oreja-Guevara C, Lafuente P, Costa Frossard L. 100 respuestas clave: planificación familiar y esclerosis múltiple. Barcelona: Ediciones Mayo; 2017.

5. Trussell J, Aiken ARA, Micks E, Guthrie KA. Efficacy, safety, and personal considerations. En: Hatcher RA, Trussell ], Nelson AL, Cates W Jr., Kowall D, Policar MS (eds.). Contraceptive technology. 21. $\stackrel{\mathrm{a}}{\mathrm{e}}$ ed. Nueva York: Ayer Company Publishers, Inc.; 2018.

6. Skouby SO. The risk of oestrogens in contraceptives. En: Briggs P, Kovacs G, Guillebaud J (eds.). Contraception. A casebook from menarche to menopause. Cambridge: Cambridge University Press; 2013. p. 42-50.

7. Odlind V, Milsom I, Persson I, Victor A. Can changes in sex hormone binding globulin predict the risk of venous thromboembolism with combined oral contraceptive pills? Acta Obstet Gynecol Scand. 2002;81(6):482-90.

8. Sitruk-Ware R. New progestagens for contraceptive use. Hum Reprod Update. 2006;12(2):169-78.

9. Agencia Española del Medicamento y Productos Sanitarios (AEMPS). Nota informativa de seguridad de los anticonceptivos hormonales combinados. Información para ciudadanos. MUH (FV), 28/2013. Madrid: AEMPS; 2013.

10. Ferreira-Filho ES, De Melo NR, Esposito Sorpreso IC, Bahamondes L, Dos Santos Simões R, Soares-Júnior JM, et al. Contraception and reproductive planning during the COVID-19 pandemic. Expert Rev Clin Pharmacol. 2020;13(6):615-22.

11. Lete I. Combined hormonal contraception and COVID-19. Eur J Contracept Reprod Health Care. 2021;26(2):128-31.

12. Mauvais-Jarvis F, Klein SL, Levin ER. Estradiol, progesterone, immunomodulation, and COVID-19 outcomes. Endocrinology. 2020;161(9):bqaa127. 